\title{
V \\ CASE OF CHANCROID OF A FINGER
}

By V. E. LLOYD, V.D. Dept., Guy's Hospital

A MALE, aged forty-four, employed on clinical work in a venereal diseases clinic for many years.

On May Ist, I935, he noticed a painful fissure between the outer border of the nail of the right middle finger and the adjoining skin. Within a few days a small tender swelling appeared at the border of the nail and some pus could be expressed.

The pain and swelling slowly increased until May 7 th, when there was a marked tender swelling with the characters of an ordinary septic inflammation extending underneath the lateral border of the nail.

He was attended to in the casualty department of the hospital, and one half of the nail was removed under gas anæsthesia.

The exposed nail bed was inflamed, but the purulent secretion released was of small volume.

An attempt next day to give treatment by local zinc chloride ionisation was followed immediately by marked pain.

By May Ioth there was further inflammatory swelling and ulceration of the exposed nail bed and adjacent tissues of the finger. The temperature was IоI $^{\circ}$; there was some toxæmia and considerable local pain. The remainder of the nail was removed under anæsthesia.

Although drainage was now free and antiseptic baths used freely, the condition progressed. On admission into a surgical ward on May I2th the finger presented an extensive foul purulent ulceration of the exposed nail bed and adjacent area. The margins of the ulcer were undermined and actively inflamed. There was considerable swelling of the distal third of the finger, but none of the hand or forearm. There was no enlargement of the epitrochlea or axillary glands.

Examination of the secretion from the ulcer for $S p$. 


\section{BRITISH JOURNAL OF VENEREAL DISEASES}

pallida was negative; the Wassermann reaction was also negative.

On May I 7 th two minute circular areas of superficial ulceration appeared in the vicinity of the proximal edge of the main ulcer. No spirochetes were found in the secretion from these small erosions. A second Wassermann reaction was negative.

A Gram-stained preparation of the secretion from the

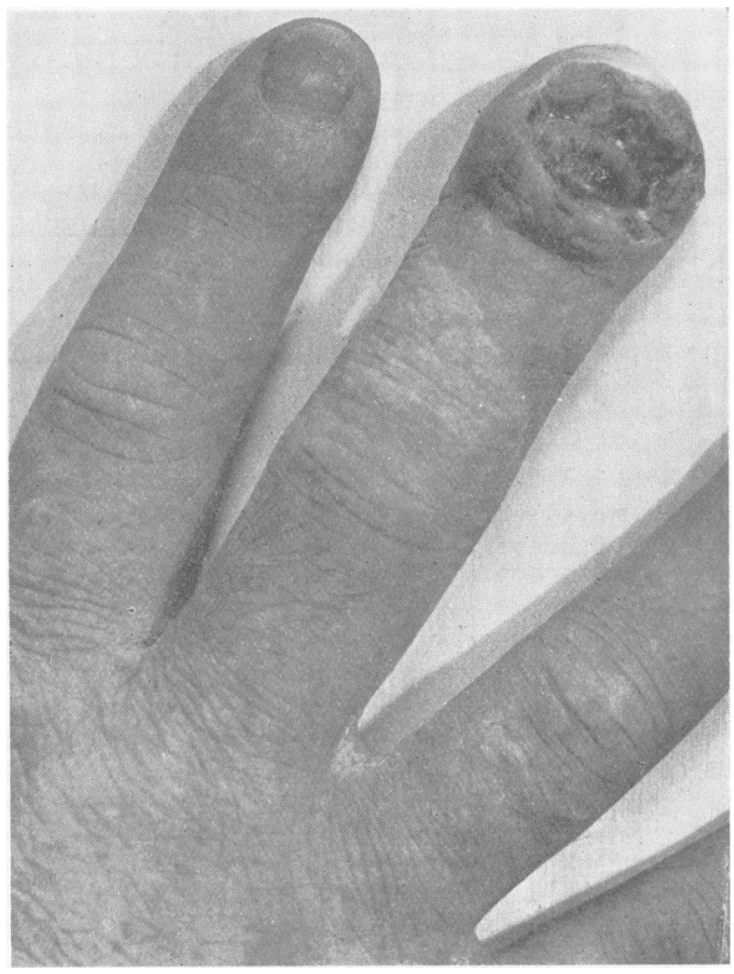

FIG. I

recent erosions showed Ducrey's streptobacillus in moderate numbers.

An intravenous injection of $\frac{1}{2}$ c.c. (IIO millions) of a vaccine of Ducrey's bacillus (Dmelcos) was given that day. There was a very rapid improvement in the amount of pain. Improvement of the inflammatory condition, slight in degree, was evident within two days. Local bathing with eusol was continued and dressings of sulphur powder. On May 23rd a second injection of Ducrey 250 


\section{CASE OF CHANCROID OF A FINGER}

bacillus vaccine $-\frac{3}{4}$ c.c. - was given. Following this, the surrounding inflammation slowly subsided, and after four or five days the ulcer margins showed signs of healing.

Frequent local bathing was now omitted and the

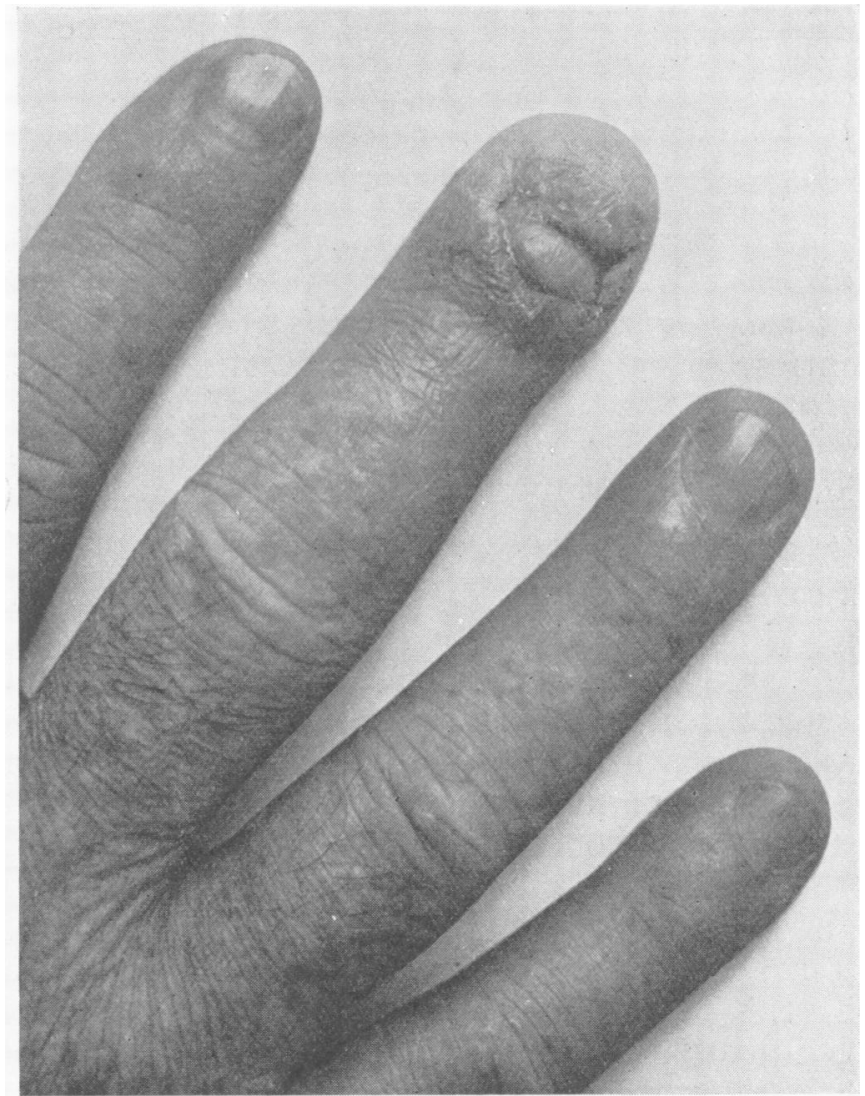

FIG. 2.

dressings changed to iodoform powder. A third injection of the vaccine-I c.c.-was given on May 3oth.

Photograph No. I shows the condition on May 30 th.

From this date onward recovery was rapid, as may be seen in photograph No. 2 taken on June IIth.

It is of interest to note that at no time has there been any glandular enlargement.

Wassermann and Kahn tests on May 24th and similar precautionary tests in early September were negative. 